\title{
Talent Evaluation System in Colleges and Universities based on Combining Internal and External Evaluation
}

\author{
J.LIU, R.YI \& Z.Wang \\ China Jiliang University, Hangzhou, China
}

\begin{abstract}
The evaluation of scientific and technological talents in colleges and universities is a very important work, but there are many problems worth studying in practical work. The problems of evaluation object, the problems of evaluation evidence, The problems of evaluation method. These all problems involve the balance and coordination of internal evaluation and external evaluation. In this paper, we using the mathematical model to analysis their respective advantages and disadvantages of the internal evaluation and external evaluation, discuss the conditions to adapt, give the implementation plan. The conclusion of this paper is that using these technology methods can reach the effective balance of internal evaluation and external evaluation, and establish combining system of talent evaluation.
\end{abstract}

KEYWORD: Talent evaluation; external evaluation; internal evaluation; Countermeasures

\section{INTRODUCTION}

Science and technology talent evaluation in colleges and universities is the value judgment on the innovative talents of this special group of people, it is to strengthen personnel work, promote the implementation of the strategy of talent powerful nation, promote the scientific and harmonious development plays a vital role in. However, there are many problems worth studying in practical work of talent evaluation in colleges and universities.

First of all, to solve the problem is who to assess problems. The evaluation may be internal colleagues, or internal assessment of experts, or the unit leadership judgment; may also be outside expert review, or anonymous peer review, etc.

Secondly, based on the evaluation problem is, that is what indicators to assess and calculate the index information from where. Is to improve yourself or tissue collection. Or expert evaluation, review, the result of the judgment, or from external audit results.

Third, is how to evaluate the problem, problem is evaluation method, what is the specific operation process, attention should be paid to the feasibility and operability, how to operate to ensure objective and fair evaluation.

Researchers have just as many of the talent evaluation and talent evaluation practitioners aware, our personnel evaluation system, especially there are many deficiencies in the evaluation of science and technology innovation talents. The existing studies pay attention to quantitative indicators, ignoring expert scientific judgment; pay attention to the neglect of the quantitative factors and qualitative factors, focusing on external achievements, while ignoring the underlying ability.

The above three problems exist inside and outside of the problem, namely the main evaluation from internal or external; evaluation of information from internal or external; how to ensure that the internal experts without human interference, how to ensure that the external experts have enough information, etc.. Internal evaluation and external evaluation involve trade-offs and effective combination problem.

This article is based on this consideration, from the point of view of suitable for China's national conditions, the establishment and external evaluation of a combination of evaluation system of innovation talents of science and technology personnel, to overcome the existing talent evaluation focusing on external quantitative indicators, ignore the internal expert judgment; the importance of external performance, despise the intrinsic ability of reality. So to improve the feasibility and reliability of the talent evaluation.

\section{LITERATURE REVIEW}

College is the innovation talents of science and technology is relatively concentrated, research personnel evaluation of University researchers 
relatively some more. Sun Dating (2009) the university teacher evaluation exists deviation, scientific research has become the core of evaluation index of research university teachers into four paradox of teacher evaluation, which is the function of the University and the diversity of evaluation index of the single, the specialized subject differences and evaluation standard of unity, scientific research difficulties and research results evaluation of the time limit, the transformation of the society and the static evaluation.

Gong Xu (2004) by three factors to discriminate between the effects of peer review of the fairness of the two types, namely the factors outside factors and review process review process itself, individual institutional factors and the appraisal process and non-system factors, a more systematic analysis of factors influencing the impartiality. Li Guanghong (2007) based on the guiding ideology and basic principles of constructing the evaluation indicator system for the analysis of high level talents, introduced the frame structure and mathematical model of evaluation index system.

Talent evaluation initially started from the talent evaluation. Personnel assessment is the basic content of modern human resource management, the major technology: a psychological test, interview and assessment center technology. Yu Jingtao (2000) a systematic review of research and application situation of domestic and foreign talent assessment, from the psychological test technology, interview techniques and evaluation center technology three aspects of talent assessment theory, method and technique route, at the same time, analysis of the current personnel assessment in the field of problems. Hu Zhenhua (2005) through the overview of theoretical basis, characteristics and development of talent evaluation technology, focus on the analysis of the characteristics of the talent evaluation technology in application.

Zhu Zhengzhou (2011) from the loss of sense of responsibility and university personnel evaluation subject talent evaluation of administrative and simplification, and re evaluation of scientific theory system, the analysis of China's science and technology talents evaluation problem of three angle light engineering technology, and discusses its cause of formation. In view of the present situation and development trend of China's science and technology talent evaluation put forward several suggestions.

Du Ying (2009) presents selection by negotiation is the core of university teacher evaluation practice out of the plight of the strategy, including the main evaluation consultation, negotiation and consensus reached evaluation criteria, the evaluation standard of consultation, evaluation of information collection and use the result of negotiation. Li Li (2010) from culture, system, knowledge and ability, the material foundation and other aspects, the implementation of the existing developmental teacher evaluation dilemma for Chinese universities to carry on the analysis, and put forward corresponding countermeasures.

On the innovation of science and technology and other senior personnel evaluation, domestic scholars mainly from the performance evaluation and index system to. Wang Lujie (2005) from two aspects of evaluation index of direct and indirect performance re design, and from the input / output angle to construct the talents of science and Technology Research Institute performance evaluation index system. Du Yulan (2011) discusses the introduction of university teaching and scientific research personnel evaluation index system. Zheng Xiucai (2002) study on University Teachers' job performance measurement method. Zhou Yin (2010) the university teacher evaluation mode from the current evaluation for teachers, refining their superiors, flat level, lower level three evaluation is the evaluation of teachers' administrative management, colleagues and students, in order to achieve the professional development of University Teachers' teaching goal and the win-win goal.

$\mathrm{Xi}$ Yuheng (2011) from the qualitative, quantitative research methods, the index system construction and its analysis method are summarized and analyzed to study the problems of how to carry on the scientific research performance of University Teachers' evaluation of domestic scholars, summarized the scholars put forward the current evaluation system deficiencies and improvement suggestions.

Just as many scholars realized that our personnel evaluation system, especially there are many deficiencies in evaluation of innovative talents in Colleges and universities of science and technology. The existing studies pay attention to quantitative indicators, ignoring expert judgment; pay attention to quantitative factors, while ignoring the qualitative factors; excessive rely on external evaluation and neglect internal evaluation. This paper analyses the internal assessment and I do not evaluate their advantages and disadvantages, discuss the adaptation conditions, gives the implementation scheme.

\section{THE KEY METHOD TO SOLVE RELATED PROBLEMS}

\subsection{The problem of internal evaluation and external evaluation}

Problem: one-sided depend on external evaluation, internal evaluation and neglect

At present, in the appraisal of talents pays too much attention to the external evaluation, internal evaluation and neglect. The number of published papers, study the evaluation guarantee external audit staff ratios in certain degree, possibility of limiting 
internal personnel relationship, go to human. But the most understanding assessment or the people around him, the information they have more comprehensive. The evaluation is based on the information to judge, one is the accuracy of the information, one is the accuracy of the judgment. The external evaluation is actually in order to improve the evaluation of non interference, and to a considerable extent, to give up the comprehensive information.

Solution: establish internal rating and external evaluation, combining the evaluation system

Internal evaluation information is comprehensive, but may be subject to interference from the interior, so the internal evaluation modeling:

$$
E_{1}(u)=E_{n}\left(I_{1}, I_{2}, \cdots I_{m}, \cdots, I_{n}, \varepsilon\right)
$$

Here $\left(I_{1}, I_{2}, \cdots I_{m}, \cdots, I_{n}\right)$ is the internal people acquire information, $\varepsilon$ is interference.

While the external evaluation is less interference, but the information is not comprehensive:

$$
E_{2}(u)=E_{x}\left(I_{1}, I_{2}, \cdots I_{m}\right)
$$

The information $\left(I_{1}, I_{2}, \cdots I_{m}\right)$ is less, but no interference.

The hypothesis of information:

$$
I_{i} \sim N\left(\mu_{i}, \sigma_{i}\right), i=1, \cdots n,
$$

Interference: $\varepsilon \sim N\left(0, \sigma_{\varepsilon}\right)$.

May be $\mu_{i}=\mu_{0}, \sigma_{i}=k \sigma_{\varepsilon}=\sigma_{0}, i=1, \cdots n$, the assumption being that the interference of internal rating may have a level $1 / k$. According to $I_{i} \sim N\left(\mu_{i}, \sigma_{i}\right), i=1, \cdots n$ the assessment can be abstracted the mean as according to random variable information $I_{i}$. To assess the accuracy of the mean standard deviation said. Then, the internal evaluation of mastering information more comprehensive, more random variables is also know its mean value decreases with the increase of variables, but because of the existence of fraud, the uncertainty will increase to a certain degree, here with an $\varepsilon \sim N\left(0, \sigma_{\varepsilon}\right)$ representation.

Then, the uncertainty of external evaluation:

$$
\sigma_{x}=\frac{\sigma}{\sqrt{m}}
$$

While the uncertainty of the internal evaluation:

$$
\sigma_{n}=\frac{\sigma}{\sqrt{n}}+\frac{\sigma}{k}
$$

When $\sigma_{n}>\sigma_{x}$ the internal interference, that is too large, should have the external evaluation. When $\sigma_{n}<\sigma_{x}$ the internal interference, little, should use the internal evaluation, because the internal master more comprehensive information.

When $\sigma_{n}=\sigma_{x}$ there is no difference between the external evaluation, internal rating. At this time:

$$
\frac{\sigma}{\sqrt{m}}=\frac{\sigma}{\sqrt{n}}+\frac{\sigma}{k}
$$

Solution: $m=\left(\frac{\sqrt{n} \bullet k}{\sqrt{n}+k}\right)^{2}$, That is to say, when the external master information $m$ Exceed $\left(\frac{\sqrt{n} \bullet k}{\sqrt{n}+k}\right)^{2}$, Use external evaluation more reliable. Otherwise, use internal evaluation. If the ratio of $m$ and internal information $n$ is $m / n=\left(\frac{\sqrt{n} \bullet k}{\sqrt{n}+k}\right)^{2} / n=\frac{k^{2}}{(\sqrt{n}+k)^{2}}$.

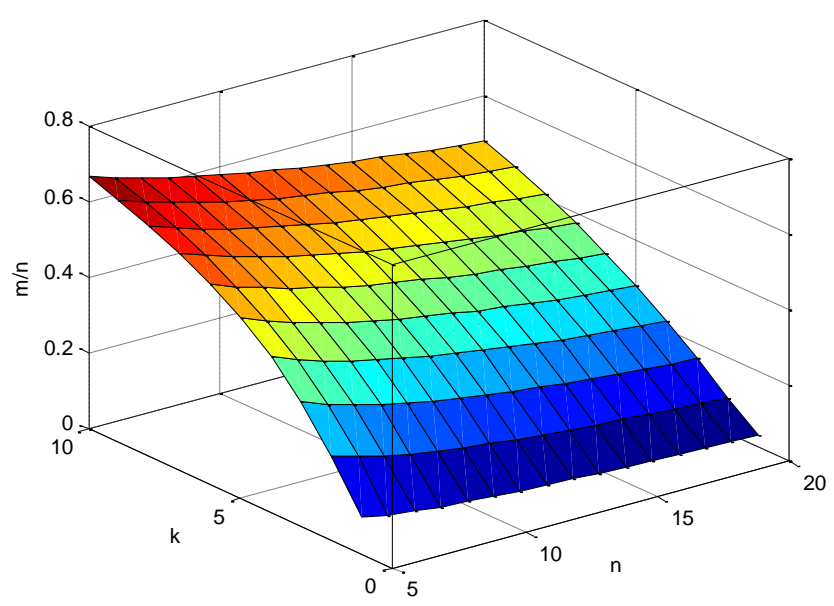

Figure 1. Relation $\boldsymbol{m} / \boldsymbol{n}$ of $\boldsymbol{n}$ and $\boldsymbol{k}$

The image above shows, the number of internal information of $\mathrm{N}$ and internal interference degree of $\mathrm{K}$ to external evaluation to grasp the information requirements of proportion. Interference is stronger, less requirement for external evaluation information. On the other hand, the internal information quantity also affect the external evaluation requires knowledge of the ratio of information, internal information more requirements for external evaluation of mastering information ratio will be lower.

\subsection{The problem of supervision and incentive evaluation committee}

Problem: Lack of mechanism of effective tracking and supervision

Talent evaluation procedure is generally: the quantitative index of the primaries, sorting, finally decided by the relevant evaluation committee vote. Effect evaluation of how no feedback and tracking mechanism. Evaluation of good and bad no 
corresponding conclusion, what is the experience a good evaluation, evaluation of bad lesson is nothing consideration. Therefore, it cannot work in the future continuous improvement.

Solution: To make objective and effective judgment to the experts, the evaluation mechanism needs to be established by evaluation experts.

The theory of the core is how to evaluate the expert evaluation results, namely, evaluation. We will set up according to the trace afterwards assessment performance, anti evaluation experts. This project aims to design effective mechanism, which will not only motivate the expert responsibility, to avoid all kinds of loopholes.

About the expert evaluation, we should not only consider the accuracy of expert judgment, but also consider the uniqueness of expert judgment. This topic plans to learn the method of information theory, the specific use similar information entropy:

$$
H(u)=\sum_{i}-p_{i} \log \left(p_{i}\right)=\sum_{i} p_{i} \log \left(p_{i}\right)^{-1}
$$

The magnitude of value of using entropy index to measure the expert judgment.

This encourages experts put forward different views, can avoid expert at the conclusion or blindly follow the views of other experts.

\section{CONCLUSION}

Talent evaluation should consider historical performance and practical working ability, to moderate the talents for the future potential; this can guarantee the fairness, strengthening incentive, facing the future. Talent evaluation should also take into account the internal evaluation and external evaluation of the combination of internal evaluation, comprehensive information, but are vulnerable to external evaluation, internal interference, less interference, but the information is not comprehensive, so to a proper balance between. Talent evaluation should have a feedback mechanism, not after the evaluation put things right once and for all, according to the late performance, moderate correction pre evaluation. Talent evaluation itself also needs to be evaluated, evaluate is to the accuracy of the evaluation, effect, the formation of a certain feedback, improve the appraisal staff's sense of responsibility, improve the accuracy of evaluation methods, improve the maximum level evaluation. Furthermore, through the technique of the proposed method in this paper can solve the specific problem. The organic combination of problems we used optimization method for solving performance ability in evaluation; solves the problem of balance of internal rating and we evaluated by statistical method. To solve the evaluation of the effect of tracking and correction problem using Bayesian method; using information theory to solve the evaluation appraisal and incentive problem itself.

\section{ACKNOWLEDGEMENTS}

This study obtained the Zhejiang province key soft science research project (2012C25083: ability combined with the performance evaluation system of the innovation talents of science and Technology) support.

\section{REFERENCES}

[1] Du Ying. 2010. Dilemma and strategy Faced with the evaluation of China's university teachers. Journal of National Academy of education administration, 2010, (09).

[2] Du Yulan, Chen Huilan. 2011. The introduction of university teaching and scientific research personnel evaluation index system study. Journal of Changzhou University (SOCIAL SCIENCE EDITION), 2011, (03).

[3] Gong Xu. 2004. Factors affecting the fairness of peer review and analysis of. studies in science of science, 2004, (06).

[4] $\mathrm{Hu}$ Zhenhua, CaiChaoying. 2005. Modern personnel assessment technique and its application. Journal of Hunan Institute of Engineering (SOCIAL SCIENCE EDITION), 2005, (01).

[5] Li Guanghong, Yang Chen.2007. High level talents evaluation index system research. progress and Countermeasures of science and technology, 2007, (04).

[6] Li Li. 2010. The implementation of developmental evaluation of teachers in Colleges and universities the plight and Countermeasures of. education and occupation, 2010, (09).

[7] Sun Dating.2009. Teacher evaluation in research university several paradoxes of. higher education of Jiangsu, 2009, (04).

[8] Wang Lujie, Hou Jian. 2005. Study on human resources development in China, the index system of performance evaluation of. talents of science and technology 2005, (01).

[9] Xi Yuheng, Zhang Dan.2011.University Teachers' scientific research performance evaluation system overview of. science and technology management research, 2011, (10).

[10] Yu Jingtao, Chen Li, Fang Suzhen. 2000. Research progress of talent assessment. soft science of health, 2000, (04).

[11] Zheng Xiucai.2002. the research on measuring method of performance of the scientific work of university teachers. the progress of science and technology and countermeasures, 2002, (12).

[12] Zhou Ying, Deng Zhongjia, Zhou Hui, Zhou Ping, 2010. The university teacher evaluation three level. idea of education theory and practice, 2010, (18).

[13] Zhu Zhengzhou, Su Weizhen, Wang Yasha. 2011. Our country science and technology talents evaluation research on the problem of. science and technology management research, 2011, (15). 